\title{
THE INTERACTION OF WEAK AND STRONG FIELDS ON THE SUN
}

\author{
HAROLD ZIRIN
}

\author{
Big Bear Solar Observatory \\ California Institute of Technology \\ Pasadena, CA 91125, USA
}

\begin{abstract}
The problems associated with unresolved flux elements are discussed. It is pointed out that the hypothesis that these fields are strong does explain various problems of solar magnetic fields, but, as all invisible phenomena, is difficult to test. It is pointed out that Zeeman splitting measurements, which give the true field, have revealed field strengths down to the lower limit of conventional spectroscopy (about 400 gauss) for years. Not one spectrogram showing larger splitting in plages or network has been produced. The power-law distribution of weak-field strengths suggests a hierarchy of field strengths, and the simple arithmetic of weak-field elements and tiny pores is on the edge of ruling out strong fields, and further improvement in resolution should do so.

Observations in the $12 \mu$ lines have shown plage fields of a few hundred gauss and broadening of the $\sigma$ components due to field variation within the field of view. These data have been explained away on the basis of height variation in the field, but the requisite transverse field strength or center-limb behavior are not observed.
\end{abstract}

\section{INTRODUCTION}

It has always been popular in astrophysics to advance outrageous hypotheses that cannot be disproved by current techniques. Often this is valuable for intellectual reasons and to stimulate new observations. Sometimes these hypotheses prove right, or partially right, such as Spitzer's galactic corona. But they are always very difficult to disprove, since the addition of a few epicycles will easily solve whatever observational disproof can be found.

The AFAS (all fields are strong) hypothesis, that all the magnetic fields in the solar photosphere are strong, say 1000 gauss, does explain some observational contradictions; but it has been extended far beyond the areas for which data has been collected, while evidence for a less radical view of the things we cannot see is ignored. Part of the problem is a desire to confirm a model, rather than seek truth. There is also a deplorable tendency to present measurements of anonymous regions, ignoring the fact that there are many levels of magnetic field, plages with fields equal to sunspots, sunspots with fields less than plages, and different levels of network and intranetwork(IN) fields. No attempt has been 
made to explain the characteristics of these well-marked and repeating regions, or why these might occur. Therefore we assume that the AFAS proponents mean that all magnetic features are composed of varying numbers of invisible elements with $1000 \mathrm{~g}$ field strength and size so we cannot see them. We assume that it is argued that apparent flux difference is due to varying number density of these elements.

\section{ARGUMENTS}

\section{PRO}

There are observational facts that the AFAS model helps to explain:

* No extended weak fields are observed. Thus all the fields seen are discrete elements, strong enough to withstand the disruptive force of photospheric turbulence.

* It is almost impossible to observe disappearance of network fields. They must be quite long lived, certainly weeks, and therefore strong.

* Line ratios suggest saturation (original Stenflo argument). as plages.

* Flux breaks off from plages, lasts forever, is probably the same strength

* The polar field near minimum is made up of magnetic elements stronger than those seen in equatorial latitudes. Although the field strength is not known, it is hard to explain this without strong fields in the network.

\section{CON}

Any model involving invisible structures, like the Emperor's new clothes, is difficult to critique because no one has ever seen it. Still, we have seen the solar magnetic fields and can therefore attack the task.

* The latest and most sensitive quiet-Sun measurements show a power-law hierarchy of field elements that fills virtually all the space and would be difficult to obtain with uniform strong elements.

* Line splittings corresponding to 1000 gauss are easy to observe. While most of the claims of 0.2 arc sec images are puffery, someone should have observed splittings of 500 gauss or more in the network or in plages if they were omnipresent.

* Line ratios are never what we expect. Explicitly, our magnetograms give no evidence for saturation right up to inner penumbra. We obtain excellent symmetric fits for the penumbral fields with different tilt angles from our magnetograms, which show no trace of saturation. There seems no question that spots can have fields of any strength above an unknown minimum, say $600 \mathrm{~g}$. The Mt. Wilson and other sunspot measurements have reported spot fields as low as $400 \mathrm{~g}$ for many years.

* 5250 spectra of plages with resolution $600 \mathrm{~g}$ show no splitting. One cannot produce $600 \mathrm{~g}$ splitting with $1000 \mathrm{~g}$ elements.

* Intranetwork and network fields show a range of field strengths which is quantitatively too great to be explained by filling factors. The smallest elements are so weak that unreasonable filling factors are required. 
* Rabin's (1992) recently published spectra do not measure splitting but fit $\mathrm{V}$ profiles, same as a magnetograph. In any event they are made in an active plage, where such fields are no surprise

* Stenflo's (1973) original measurements, and everything since, have been done on spectrographs with narrow slits and sensitive lines. The videomagnetograph at BBSO uses an $0.25 \AA$ filter on a fairly broad line. As we shall see below, there is no sign of saturation between IN and network fields, as should occur if there are strong fields in all elements.

* Measurements in the $12-\mu$ line do not reveal strong fields. Deming et al. (1988) obtained 12- $\mu$ spectra showing penumbral splitting of 600 gauss, as do our spot videomagnetograms. Zirin and Popp (1989) found network fields below $200 \mathrm{~g}$, with no evidence for field divergence to that level.

* "Invisible spots" have a definite non-zero size. Combining with flux measurements on magnetograms leads to small field strengths. White-light images show spots to be homogeneous down to a few tenths of an arc sec. It is impossible to fit the observed spots with strong fields because absorption cannot exceed $100 \%$. The incompatibility between elements that can have very strong fields but cannot absorb more than $100 \%$ causes difficulties for the AFAS model.

* IN fields are definitely weaker than network fields since invisible spots are never seen outside network.

* Zirin (1992) has shown the existence of 3500 -g fields, and also 1500 -g outside of sunspots. Since these cannot be formed out of the 1000-gauss elements, a second kind must exist. In fact, the nature of sunspots is ignored in AFAS. Given the undeniable existence of these fields, how do they transform to $1000 \mathrm{~g}$ fields?

\section{DETAILS}

The arguments in favor of AFAS are all indirect measurements of unknown field configurations. Stenflo (1973) measures fields of less than a hundred gauss in an unknown Sun-center field (we looked at it and found a mixture of quiet Sun and network), and decides they are a thousand gauss. Rabin measures 1000 gauss (by a magnetograph technique) in a highly active plage and Stenflo decides that is the intensity of the weak fields.

If a single spectrogram in the network showed the large splittings required, one could readily agree with the AFAS model. But for some reason, only indirect measurements or measurements in plages are presented. Measurements in the $12-\mu$ lines were supposed to reveal the true fields, because they show the complete Zeeman pattern. When no fields above 200 gauss were found, epicycles were added to the model, and it was decided that the fields were diverging from the base and were already weak at the height of formation of these lines. Measurements of the lines by Zirin and Popp (1989) contradict this conclusion: if the fields diverge, a sizable unshifted $\pi$ component would appear, but none was found. Examination of NIXT X-ray images shows rather small divergence, about 40 deg or less, not enough to reduce 1000 gauss to 7 gauss by the $500 \mathrm{Km}$ altitude of formation of the lines.

The measurement of the center-limb variation of the fields should shed some light on this question. The longitudinal fields should show a $\cos \theta$ falloff due to projection, plus a decrease due to divergence (because we look about one scale 
height higher) if present. We have found a general $\cos \theta$ falloff at Big Bear for the polar field. However, when the polar field is strong, we see clear divergence. Thus one cannot discount the fields measured by the $12-\mu$ line as weakened by canopy divergence.

The relative strength of magnetic fields in plages, spots and network illustrates the problems that have been brushed aside. A realistic model of how the strong fields might comprise the typical plage, with fairly uniform field of the order of 300 gauss over extended areas, has not been made. Since the filling factor for canonical 1000-gauss elements would be only 3, a spectrogram with high resolution would show some splitting where the invisible fields might clump. None is seen, nor is much fluctuation seen in the fairly regular patterns.

\section{INVISIBLE SPOTS}

The transition between the invisible field elements and the smallest detectable spots is also not touched on by the strong-field model. Originally the detection of "invisible spots" was a test of the AFAS model; if the network fields were so strong, why didn't we see spots? We (Zirin and Wang 1992) found spots, but they were the wrong size, too big (1-2 arc sec) and too weak $(200 \mathrm{~g})$, with darkening about $10 \%$. Allowing for MTF and scattered light suggests about $30 \%$ real darkening. This means a minimum of $30 \%$ of the tiny spots should be filled with $100 \%$ absorbing spots. But if the truly invisible field elements absorb less than $100 \%$, as is likely, the field in these tiny spots would exceed the $200-300$ gauss measured value. Please remember that our broad slit magnetograph is well calibrated and does not saturate.

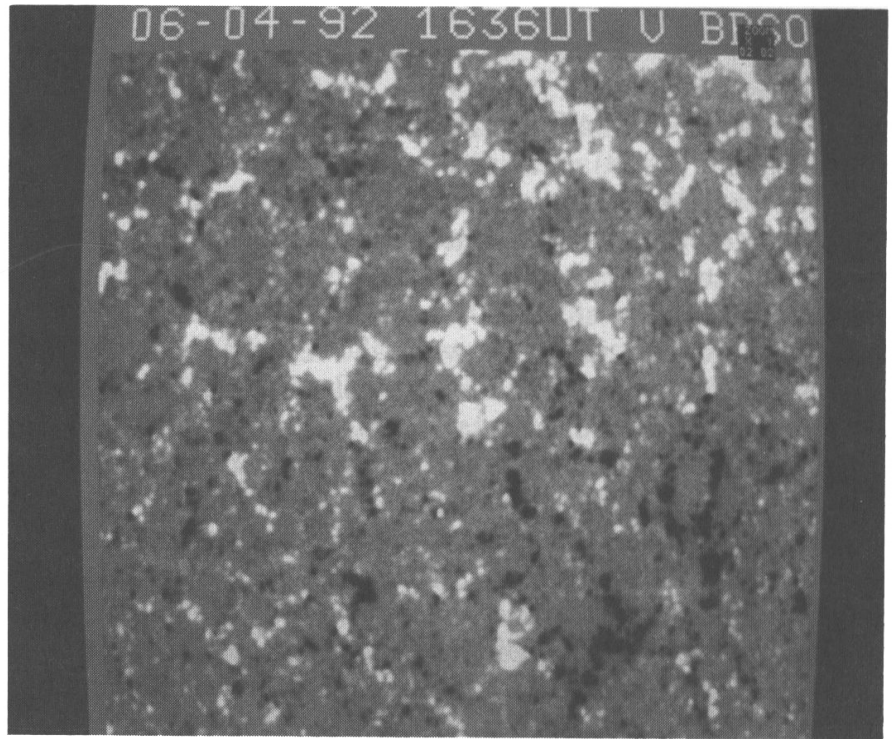

FIG. 1. Quiet Sun magnetogram; the weakest elements observed are 7 gauss and $1.3 \times 10^{17} \mathrm{Mx}$. An $\mathrm{H} \alpha$ filament winds diagonally across the frame separating mostly negative polarity at lower right from mostly positive on upper left. 
The origin and evolution of magnetic fields can shed light on their properties even if we can't see the fields themselves. One of the most puzzling aspects of spot formation is the ability of small spots to metamorphose into plage with no apparent effect on the magnetogram. It is hard to explain how 10 tiny spots, which absorb $100 \%$, can suddenly turn into bright plage. As pointed out above, while one can explain emission with filling factors, one cannot do the same with absorption, as an invisible sunspot cannot absorb more than $100 \%$ of the radiation coming from above. Thus if 100 invisible spots, each 0.1 arc sec across, merge to form a 1 arc sec spot, we will need to bring them in from a much larger area, roughly in the ratio of the spot to plage field. And, of course, a big spot forming in an EFR displays converging fields, but in far fewer number than required by the AFS model. Of course the formation of 3000-gauss sunspots is not provided for in the AFAS model, nor the equilibrium of multiple flux tubes packed close together.

\section{QUIET SUN FIELDS}

The intranetwork fields were discovered by Harvey many years ago, and the first detailed observations presented by the author (Zirin, 1986). But my learned colleague claims to have discovered them last year. While the fact that he has seen the light is good, it is a little bit like denying the moons of Jupiter and then discovering them.

A weak network covers the Sun at all times, whether or not there have been spots present. This network is weaker when there haven't been spots, so we deduce the fields are weaker than those in spots. Inside the network are the even smaller IN fields. So far as I know, our colleagues have never observed these fields, with the exception of the original work (Stenflo, 1973). Instead we have observations of unknown plages, never illustrated. In the AFAS model, this variation depends on the number density and total number of EIS (elemental invisible sunspots) in each flux element.

Figure 1 shows a very sensitive magnetogram, with a threshold about 7 gauss for the weakest detectable elements that can be seen on two separate VMG's. A histogram shows the pixel number for which the field exceeds 7 gauss to be about $50 \%$ of the overall area. Thus most of the surface is covered with distinct magnetic elements (I assume we have not yet reached the limit). This distribution holds very well; through the entire day, the standard deviation of the field about null was 42 , about 14 gauss. Our calibration on the 16-bit images is 0.012 gauss $/$ pixel, with a range \pm 3000 pixels, or $.2813 \mathrm{~g} / \mathrm{pixel}$ on a \pm 128 display, which is saturated by a 30 -gauss field. The smallest real elements are about 25 pixel values or 7 gauss above the background (this is the lowest level at which the element is visible on several successive frames). The apparent size of these elements is 2 arc sec, so the flux is $1.3 \times 10^{17} \mathrm{Mx}$ in these elements, about 3 times smaller than the weakest elements measured by Rüedi et al. If the field strengths were 1000 gauss, the filling factor would be 12 , which does not rule out the AFAS model. Another important factor is the formation and evolution of fields. The value quoted is considerably greater than that found by Wang et al. (1985), and by Zirin (1986) based on their data, but there were obvious mistakes in the Wang et al. calibration. The present data is based on line slope calibration carried out by Mr. Glenn Eychaner, which we feel is reasonably accurate, and has been compared with other magnetographs. In the 
AFAS model a single 1000-gauss flux element would then be $100 \mathrm{Km}$ across. Detection of weaker elements will make the AFAS model untenable, as the scale is then below the radiation mean free path.

Figure 2 is a $\log$-log plot of the distribution of pixel values in one of our best magnetograms. Note that it gives pixel values, not element values. The largest field strength here is 36 gauss, and the distribution is a power law with exponent -1.4 up to the smallest elements. Because the power law extends well below the threshold value at which we can identify a field element on several frames, we are led to believe that further improvement in the magnetograph will lead to detection of weaker elements, and the movie suggests that the intranetwork area is filled completely with this hierarchy of fields. Histograms of the distribution show that $50 \%$ of the area lies above the 7-gauss level. Unfortunately we do not have software to identify elements, but mostly these must be in the IN and network fields.

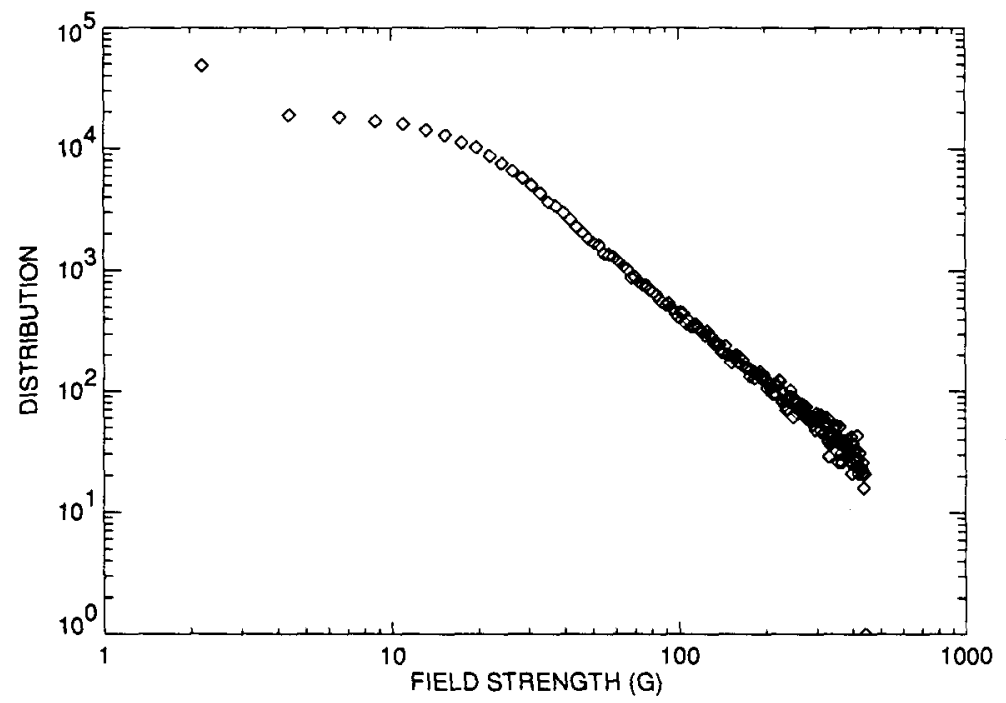

FIG. 2 Distribution of pixels in absolute intensity in the magnetogram; the distribution closely follows a power law with exponent -1.4 .

When we examine the 8-hr movie of this data, we see that the IN elements move rapidly, but not always toward the cell walls. The network elements change gradually in shape, but do not break up or form new ones. The number of disappearing elements outnumbers those forming, yet the field distribution is constant; therefore larger elements must form than disappear. Finally, we must remember that these elements only represent the intersection of field lines with the surface, and their behavior above or below is unknown.

\section{CONCLUSION}

Well, OK, Zirin, but what is your explanation?

I do not see the reason to require fields of a fixed and magic strength. Obviously they must be smaller than what we see, because the resolution of our 
magnetographs is limited. But the fields we do see exhibit a great range of intensity, and it is silly to believe that this is due to the density of element fields alone. The fact that fields form and disappear by reconnection and merging means that the $100 \mathrm{~g}$ fields would have to go to zero, and field theory forbids discontinuous jumbos in field strength, because derivatives enter into the Maxwell equations. Because the sub-surface fields must be stronger, it is not necessary for the surface fields to be strong enough to hold them together. As one watches the fields appear and disappear, one sees that reality is much more interesting than a world of quantized flux elements.

One of the principal arguments adduced by our learned colleague is that everyone agrees with him that the fields are strong. It reminds me of a review paper written in the early sixties which stated that everyone, with the exception of a few die-hards in Boulder (Billings, Roberts and myself) believed that the ionization theory gave the correct value for the coronal temperature. A few months later the ionization theory fell, with the introduction of dielectronic recombination, and the coronal temperatures rose to that given by the Doppler data.

Despite these complaints, we should all be grateful to Prof. Stenflo for these ingenious ideas that have produced such interesting controversy and stimulated new research in the field.

Our work at Big Bear has been supported by ONR grant N00014-89-J1069, NASA grant NAGW-1972 and NSF grant ATM-9122023.

\section{REFERENCES}

Deming, D. et al.: 1988, Ap. J., 333978

Rabin, D.: 1992, Ap. J., 391832

Stenflo, J. O. 1973, Solar Phys., 3241

Wang, J., H. Zirin, and Z. Shi: 1985, Solar Phys., 98241

Zirin, H.: 1986, Aust.J.Phys. 38961

Zirin, H.: 1992, BBSO \#344, submitted to Ap. J.

Zirin, H. and Popp, B.: $1989, A p$. J., 340, 571

Zirin, H. and Wang, H.: 1992, Ap. J., 385 L27 